\title{
FTY720 Attenuates Neuropathic Pain after Spinal Cord Injury by Decreasing Systemic and Local Inflammation in a Rat Spinal Cord Compression Model
}

\author{
Kazuyoshi Yamazaki, ${ }^{1}$ Masahito Kawabori, ${ }^{1,2}$ Toshitaka Seki, ${ }^{1}$ Soichiro Takamiya,, Takahiro Tateno, ${ }^{3}$ \\ Kotaro Konno, ${ }^{4}$ Masahiko Watanabe, ${ }^{4}$ and Kiyohiro Houkin ${ }^{1,2}$
}

\begin{abstract}
Neuropathic pain severely impairs rehabilitation and quality of life after spinal cord injury (SCI). The sphingosine-1phosphate receptor agonist, FTY720, plays an important protective role in neuronal injury. This study aims to examine the effects of FTY720 in a rat acute SCI model, focusing on neuropathic pain. Female rats with SCI induced by 1-min clip compression were administered vehicle or $1.5 \mathrm{mg} / \mathrm{kg}$ of FTY720 $24 \mathrm{~h}$ after the injury. Using the mechanical nociceptive threshold test, we monitored neuropathic pain and performed histological analysis of the pain pathway, including the $\mu$ opioid receptor (MOR), hydroxytryptamine transporter (HTT), and calcitonin gene-related peptide (CGRP). Motor score, SCI lesion volume, residual motor axons, inflammatory response, glial scar, and microvascular endothelial dysfunction were also compared between the two groups. FTY720 treatment resulted in significant attenuation of post-traumatic neuropathic pain. It also decreased systemic and local inflammation, thereby reducing the damaged areas and astrogliosis and resulting in motor functional recovery. Whereas there was no difference in the CGRP expression between the two groups, FTY720 significantly preserved the MOR in both the caudal and rostral areas of the spinal dorsal horn. Whereas HTT was preserved in the FTY720 group, it was significantly increased in the rostral side and decreased in the caudal side of the injury in the vehicle group. These results suggest that FTY720 ameliorates post-traumatic allodynia through regulation of neuroinflammation, maintenance of the blood-brain barrier, and inhibition of glial scar formation, thereby preserving the connectivity of the descending inhibitory pathway and reducing neuropathic pain.
\end{abstract}

Keywords: allodynia; inflammation; FTY720; glial scar; spinal cord injury

\section{Introduction}

S PINAL CORD INJURY (SCI) occurs when the structures surrounding the spinal cord are damaged by fractures, dislocation, expansion, or compression. Although contusion-compression injury mostly causes incomplete damage of the spinal cord, SCI causes devastating sequelae, such as severe motor, sensory, and autonomic dysfunction. More than 1 million patients worldwide suffer from paresis caused by SCI. ${ }^{1}$ In addition to paraparesis, treatment of neuropathic pain at or below the level of injury is one of the typical medical unmet needs of patients with SCI. Approximately $40-60 \%$ of these patients are reported to have neuropathic pain, and half of these are reported to be moderate-tosevere cases. ${ }^{2,3}$ Pain is deeply related to the deterioration of physical, mental, and social functions and is thought to cause dysfunction beyond restricting rehabilitation by interfering with sleep and daily life and therefore worsens overall quality of life. ${ }^{3-5}$ Many pharmacological therapies have attempted to reduce the damage caused by SCI in the past, but no single therapy has been able to dramatically reverse serious paralysis and sensory disturbances. ${ }^{6}$

FTY720 is a novel immunomodulator which acts as a sphingosine-1-phosphate (S1P) receptor agonist and has recently been approved for treatment of multiple sclerosis (MS) by the U.S. Food and Drug Administration. ${ }^{7}$ Effects of FTY720 mainly include systemic lymphopenia and inhibition of local inflammatory responses. However, recent reports have shown that it can also reduce glial scar formation by modulating activated astrocytes and reorganizing the inadequate local environment. ${ }^{8-11}$ There are several reports showing the efficiency of FTY720 for SCI, although most of

\footnotetext{
${ }^{1}$ Department of Neurosurgery, Graduate School of Medicine, ${ }^{2}$ Department of Neurological Cell Therapy, Hokkaido University Hospital, ${ }^{3}$ Department of Hematology, Graduate School of Medicine, ${ }^{4}$ Department of Anatomy and Embryology, Graduate School of Medicine, Hokkaido University, Sapporo, Hokkaido, Japan.

(C) Kazuyoshi Yamazaki et al., 2020; Published by Mary Ann Liebert, Inc. This Open Access article is distributed under the terms of the Creative Commons Attribution Noncommercial License (http://creativecommons.org/licenses/by-nc/4.0/) which permits any noncommercial use, distribution, and reproduction in any medium, provided the original author(s) and the source are credited.
} 
them focus only on motor recovery. ${ }^{9,12,13}$ The aim of this study is to evaluate the protective effects of FTY720 in an acute phase clinically relevant SCI model, focusing on the attenuation of neuropathic pain.

\section{Methods}

\section{Experimental ethics}

All experimental protocols were approved by the Animal Studies Ethical Committee at the Hokkaido University Graduate School of Medicine (reference no.: 17-0066). All procedures used in the present study were performed in accordance with the Institutional Guidelines for Animal Experimentation and the Guidelines for Proper Conduct of Animal Experiments of the Science Council of Japan.

\section{Experimental animals}

Wild-type 8- to 10-week-old female Sprague-Dawley rats (CLEA Japan, Inc., Tokyo, Japan), weighing 250-300 g, were used in this study. Female rats were used for better urination management after the SCI. Animals were housed in a controlled environment $\left(25^{\circ} \mathrm{C}, 50 \%\right.$ humidity, and 12-h light-dark cycle) and were allowed free access to food and water. All experimental animals were randomly divided into vehicle-treated $(n=15)$ and FTY720-treated $(1.5 \mathrm{mg} / \mathrm{kg} ; n=15)$ groups.

\section{Spinal cord injury model}

Thoracic SCI was induced by a 1-min compression using a modified aneurysm clip, which was designed by the authors and manufacturers (Mizuho, Tokyo, Japan) as previously reported, with a slight modification. ${ }^{14-16}$ The face of the clip blade was smoothened to apply equal pressure on the spinal cord, and the closing force was set to $30 \mathrm{~g}$. Rats were anesthetized using isoflurane at an initial and maintenance concentration of $4.0 \%$ and $2.0 \%$, respectively, in $70 \% \mathrm{~N}_{2} \mathrm{O}$ and $30 \%$ $\mathrm{O}_{2}$ gas through a facial mask. Rats were placed in the prone position, and using the T2 spinous process as a landmark, laminectomy was performed at the T6 and T7 vertebral segments. The aneurysm clip was then applied extradurally to fully compress the spinal cord at the T6 level for $1 \mathrm{~min}$. After closing the skin, the animals were allowed to recover on heated towels until they were fully awake. Rats with a relatively low motor deficit (Basso-Beattie-Bresnahan [BBB] score of $<7) 24 \mathrm{~h}$ after the insult were excluded from the subsequent studies. Bladders were manually pressed for urination three times daily until spontaneous reflexive bladder control was regained. ${ }^{17}$

\section{Administration of FTY720}

FTY720 or saline (vehicle) was injected intraperitoneally $24 \mathrm{~h}$ after the SCI. Rats in the FTY720 group received $1.5 \mathrm{mg} / \mathrm{kg}$ of FTY720 (Cayman Chemical, Ann Arbor, MI) diluted in saline, whereas the control group received the same volume of plain saline.
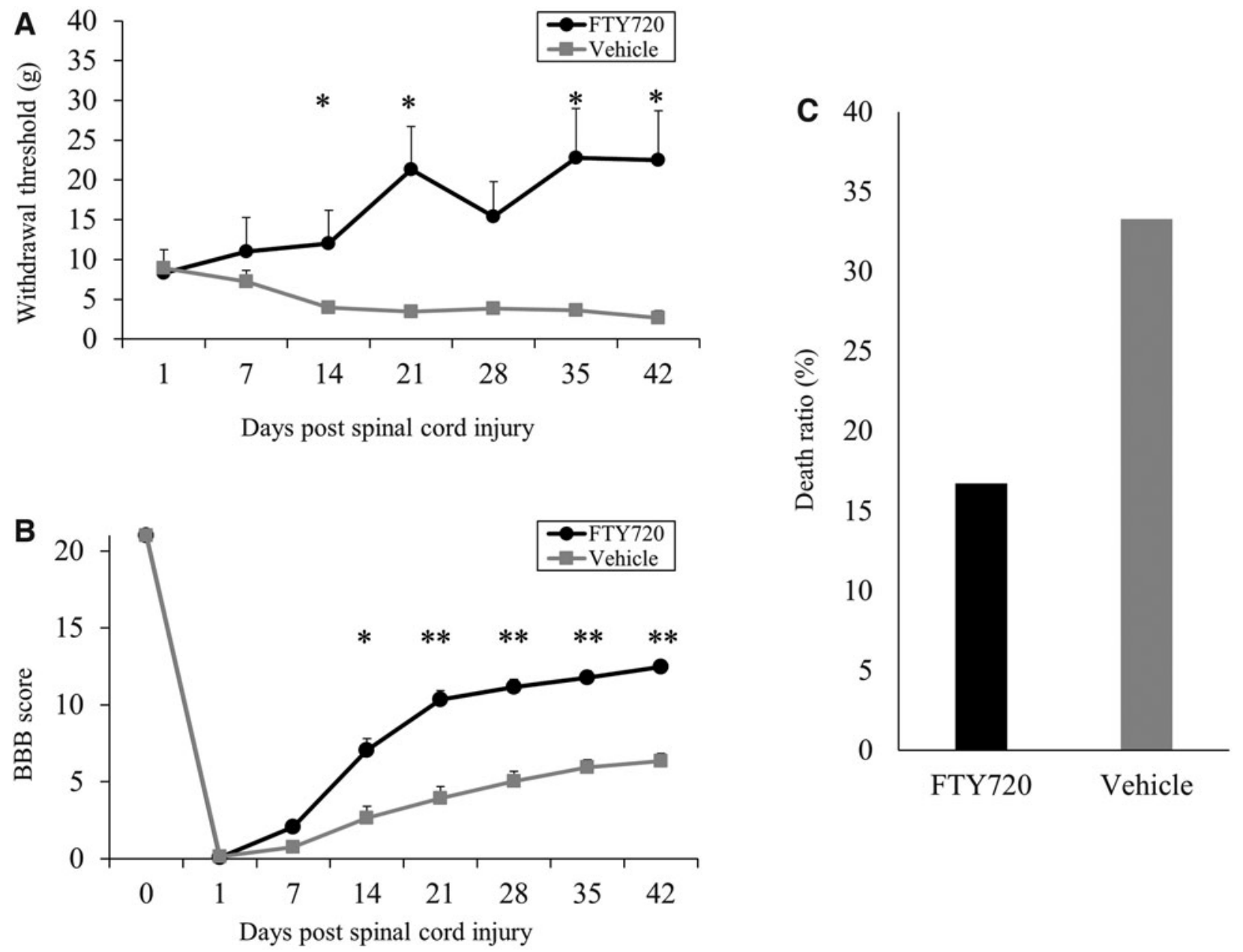

FIG. 1. FTY720 attenuates neurological deficit and reduces mortality. FTY720-treated animals $(n=13)$ show significant $($ A) reduction in the threshold of neuropathic pain assessed using the von Frey monofilament test $(* p<0.05)$ and $(\mathbf{B})$ improvement in motor recovery as estimated using the Basso-Beattie-Bresnahan score when compared to vehicle-treated animals $(n=10 ; * * p<0.01)$. (C) FTY720 treatment decreases the overall mortality ratio after the SCI. BBB, Basso-Beattie-Bresnahan score; SCI, spinal cord injury. 


\section{Neurological score}

All neurological assessments were performed in a blinded fashion by two independent observers on days $7,14,21,28,35$, and 42 postinjury. The von Frey monofilament test (Touch Test Sensory Evaluator Kit; Stoelting Co., Wood Dale, IL) was performed to evaluate mechanical allodynia as previously reported. ${ }^{18,19}$ Briefly, rats were placed on a rubber mat, and a set of von Frey hair was used to test the cutaneous pain threshold in response to graded mechanical pressure, ranging from 0.02 to $300 \mathrm{~g}$, on the dorsal surface of the trunk, mainly at the base of the tail. Only stimuli that induced consistent behavior of avoidance and vocalization over a relatively large skin area were considered as the withdrawal thresholds. A single trial consisted of 12 applications of the von Frey filament, applied once every 2-3 sec.

The BBB (hindlimb locomotor test) score was used to evaluate the neurological motor deficits as previously reported. ${ }^{15,18-20}$ Hindlimb locomotor function was evaluated separately for $5 \mathrm{~min}$ in the open field, and values were averaged to obtain individual scores. All data from animals that died during the evaluation period were excluded from the analysis.

\section{Evaluation of blood lymphocytes}

Flow cytometry using fluorescent-activated cell sorting (FACS) was performed on the third day after the SCI to assess systemic inflammation by counting lymphocytes in the peripheral blood during the acute phase of the injury, as previously described. ${ }^{21}$ Approximately $30 \mu \mathrm{L}$ of peripheral blood was collected on the third day of injury from the tail vein, and erythrocytes were lysed using
RBC lysis buffer (BD Pharmingen, San Diego, CA). Using flow cytometry (FACS Canto; BD Biosciences, San Jose, CA), dead cells were excluded based on 4',6-diamidino-2-phenylindole positivity (Dojindo Laboratories, Kumamoto, Japan). Next, CD3 positive $T$ cells were identified using an anti-CD3 (PE anti-rat CD3 antibody; BioLegend, San Diego, CA) antibody, and their proportion in the peripheral blood was measured.

\section{Evaluation of the spinal cord injury lesion volume}

The volume of the SCI-induced lesion was evaluated 42 days after the SCI using hematoxylin eosin (HE) and Luxol fast blue (LFB) staining for the detection of neuronal cells and myelin, respectively. ${ }^{15,22}$ Rats were deeply anesthetized using $4.0 \%$ isoflurane in $\mathrm{N}_{2} \mathrm{O} / \mathrm{O}_{2}$ (70:30) to prevent pain and discomfort at the time of euthanization. Animals were perfused transcardially with $50 \mathrm{~mL}$ of physiological saline, followed by $150 \mathrm{~mL}$ of $4 \%$ paraformaldehyde. The thoracic spinal cord (T2-T11) was removed, post-fixed in the same fixative overnight, and immersed in $20 \%$ sucrose in phosphate-buffered saline at $4^{\circ} \mathrm{C}$ for $2-3$ days. The blocks were then embedded in optimal cutting temperature compound (Tissue-Tek; Sakura Finetechnical Co., Tokyo, Japan), and $10-\mu \mathrm{m}$-thick sagittal sections or $4-\mu \mathrm{m}$-thick transverse sections were mounted onto poly-L-lysine-coated slides (Matsunami, Tokyo, Japan) for subsequent immunostaining.

Length and volume of the lesions were calculated by the method described previously. ${ }^{15,22,23}$ Briefly, a sagittal section of the spinal cord was used to calculate the lesion length. The distance from the epicenter of the lesion to the most rostral and caudal points
A

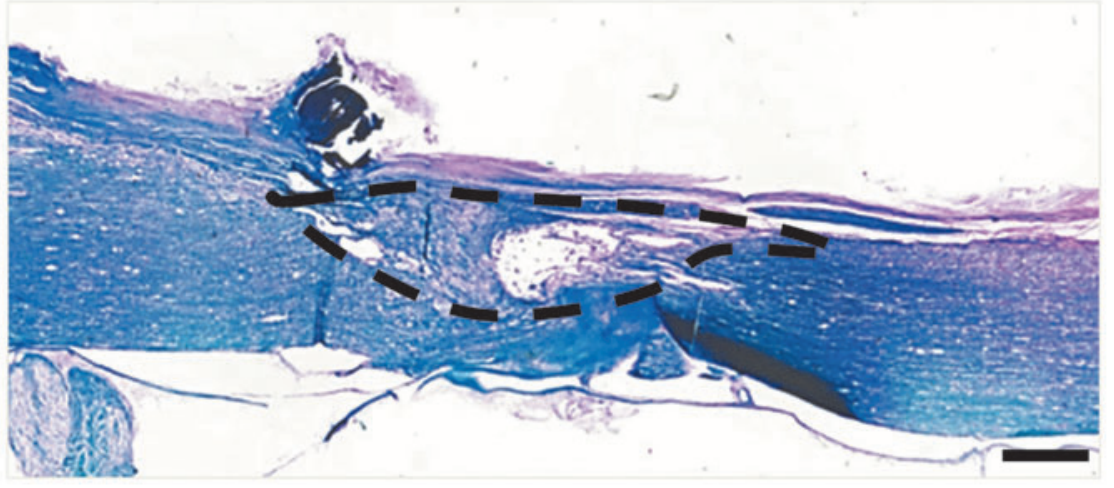

B

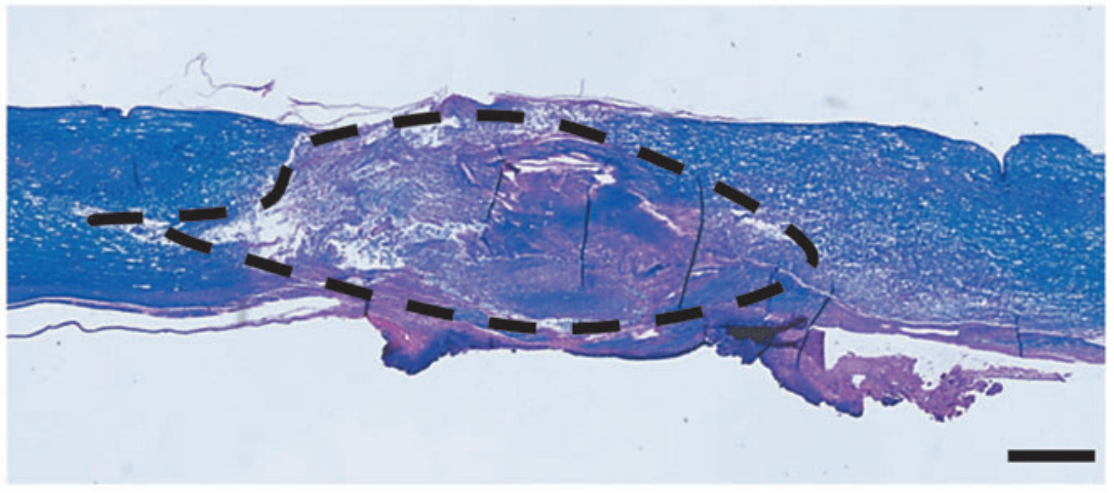

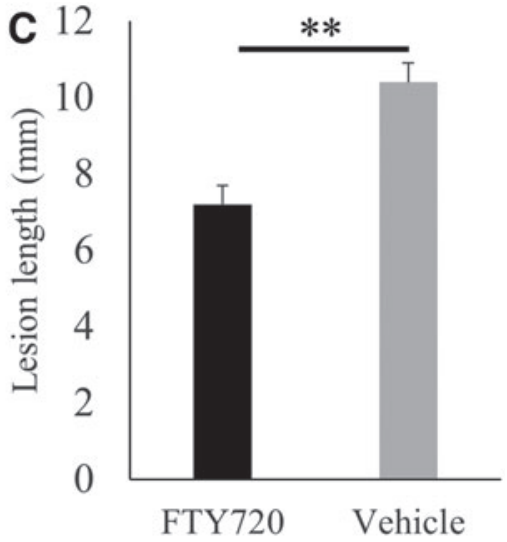

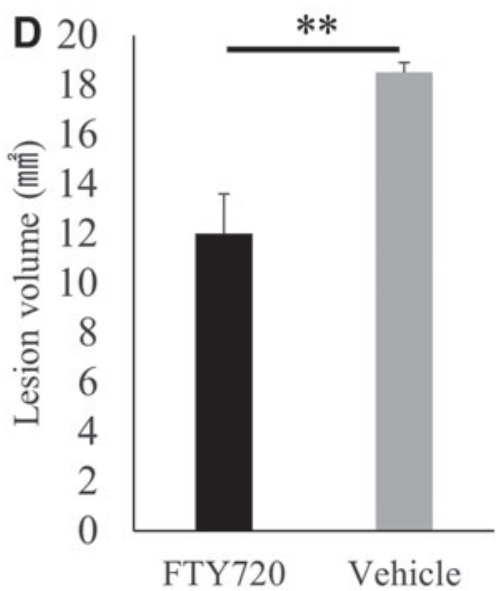

FIG. 2. Effect of FTY720 on SCI lesion size. HE and LFB staining of sagittal spinal cord sections 42 days after SCI to assess demyelination. The representative images here show a smaller lesion area in the (A) FTY720-treated group compared to that in the (B) vehicle-treated group. A significant reduction in the $(\mathbf{C})$ length and (D) volume of the lesion can be seen in the FTY720-treated group $(* * p<0.01)$. HE, hematoxylin and eosin; LFB, Luxol fast blue; SCI, spinal cord injury. 
determined by the absence of LFB staining was semiautomatically calculated using a BZ-X analyzer (Keyence, Osaka, Japan). Lesion volume was estimated by adding the area of damage from multiple sections determined using LFB staining and calculated using the following equation ${ }^{23}$ : Lesion volume $\left(\mathrm{mm}^{2}\right)=\pi \mathrm{D}^{2}(\mathrm{H} 1+\mathrm{H} 2) / 6$, where $\mathrm{H} 1$ is the lesion length from the epicenter to the rostral end, $\mathrm{H} 2$ is the lesion length from the epicenter to the caudal end, and D is the diameter of the epicenter.

\section{Immunohistochemistry}

Inflammation and blood-brain barrier integrity were evaluated 7 days after the SCI ( $n=5$ for each evaluation). Ionized calcium binding adaptor molecule 1 (Iba1) and CD68 expression were evaluated to assess neuroinflammation. Sections were treated for $1 \mathrm{~h}$ at room temperature with anti-Iba1 (1:1500, 019-19741; Wako Co., Tokyo, Japan) and anti-CD68 (1:1000, MCA341GA; Bio-Rad Laboratories, Hercules, CA) antibodies. After the first antibody incubation, sections were treated with the Histofine ${ }^{\circledR}$ Simple StainTM Rat MAX PO (Nichirei Biosciences Inc., Tokyo, Japan) for $1 \mathrm{~h}$. The 3,3'-diaminobenzidine (DAB) chromogen of the DAB substitute kit (Nichirei Biosciences) was applied for 10-30 sec to obtain a chromogenic signal in accordance with the manufacturer's instructions. Images of the peri-damaged lesions $5 \mathrm{~mm}$ caudal and $5 \mathrm{~mm}$ rostral from the epicenter of the lesion were obtained. Five non-overlapping lesions were randomly selected, and positive cells were semiquantitatively counted using an automated cell counter (BZ-X Analyzer; Keyence).

Blood-brain barrier integrity was quantitatively assessed by evaluating the fibrin leakage around blood vessels as described previously. ${ }^{24-26}$ A transverse section $5 \mathrm{~mm}$ caudal from the lesion core was used for the evaluation, and five blood vessels of approximately $50 \mu \mathrm{m}$ in diameter were randomly selected near the center of the section. Fibrinogen leakage around the selected blood vessels was measured using antifibrinogen gamma chain (1:100; Proteintech Group Inc, Rosemont, IL), followed by DAB staining. The area of fibrinogen leakage was then evaluated using an automated area counter (BZ-X Analyzer; Keyence).

Post-traumatic glial scar formation was evaluated based on the expression of the glial fibrillary acidic protein (GFAP). Using an antiGFAP antibody (dilution 1:200; BD Biosciences, Franklin Lakes, NJ), GFAP expression was assessed in sagittal sections. Sensoryrelated synapse and neurons were evaluated using the anti- $\mu$ opioid receptor $(\mathrm{MOR})^{27}$ (dilution 1:200; Abcam, Cambridge, UK), antihydroxytryptamine transporter (HTT), ${ }^{28}$ and anticalcitonin generelated peptide (CGRP; C8198; dilution, 1:8000; Sigma-Aldrich, St. Louis, MO) antibodies, as previously reported. Areas of MOR-, HTT-, and CGRP-positive cells in the spinal dorsal horn $1 \mathrm{~mm}$ rostral and caudal from the epicenter of the injury were semiautomatically counted in a transverse section. ${ }^{29}$

\section{Quantification of the labeled dorsal corticospinal tract}

To trace the dorsal corticospinal tract (dCST), animals (3 each from both groups) were anesthetized 42 days after the SCI, as described above. They were fixed in a stereotactic apparatus (Model DKI-900; David Kopf Instruments, Tujunga, CA), and the midline incision wound was reopened. The needle of a $10-\mu \mathrm{L}$ Hamilton syringe was positioned $10 \mathrm{~mm}$ rostral to the center of injury and inserted $1.5 \mathrm{~mm}$ into the spinal cord from the surface of the dura mater. Fluoro-Ruby (FR; $2 \mu \mathrm{L} ; 10,000$ molecular weight, D-1817; Molecular Probes, Eugene, OR), a fluorescent axonal tracer, was injected into the spinal cord $0.5 \mathrm{~mm}$ lateral to the midline on both sides over a period of 3 min, using an automatic microinjection pump (Model KDS-310; Muromachi Kikai Co., Tokyo, Japan), as previously reported. ${ }^{30}$ The needle was left in place for 3 min and then withdrawn slowly to minimize leakage of the tracer. After the injection, the wound was closed, and animals were housed.
To visualize the residual dCST axons, rats were euthanized 5 days after the FR injection, and transverse sections of the spinal cord $10 \mathrm{~mm}$ caudal from the injury site were observed. Given that the dCST is known to exist within the ventral one-third of the dorsal funiculus in rats, the number of FR-labeled axons in this area was counted bilaterally. ${ }^{30,31}$

\section{Statistical analysis}

All the data were collected and analyzed by investigators in a blinded manner. The data have been presented as mean \pm standard deviation. All statistical analyses were performed using JMP Pro software (version 14; SAS Institute Inc., Cary, NC). Differences between the two groups were compared using an unpaired $t$-test or the Mann Whitney $\mathrm{U}$ test, and $p<0.05$ was considered statistically significant.

\section{Results}

FTY720 improves allodynia and functional motor recovery and reduces mortality

FTY720-treated animals showed significant improvement in the threshold of neuropathic pain as monitored by the von Frey monofilament test (Fig. 1A). Further, these animals showed significant functional motor recovery and lower mortality when compared to vehicle-treated animals ( $p<0.05$; Fig. 1B,C).

\section{FTY720 decreases the size of spinal cord injury lesions}

FTY720 treatment significantly decreased the length and volume of the injury lesion, as determined by the absence of LFB staining, in sagittal sections 6 weeks after the SCI $(p<0.01 ;$ Fig. 2$)$.

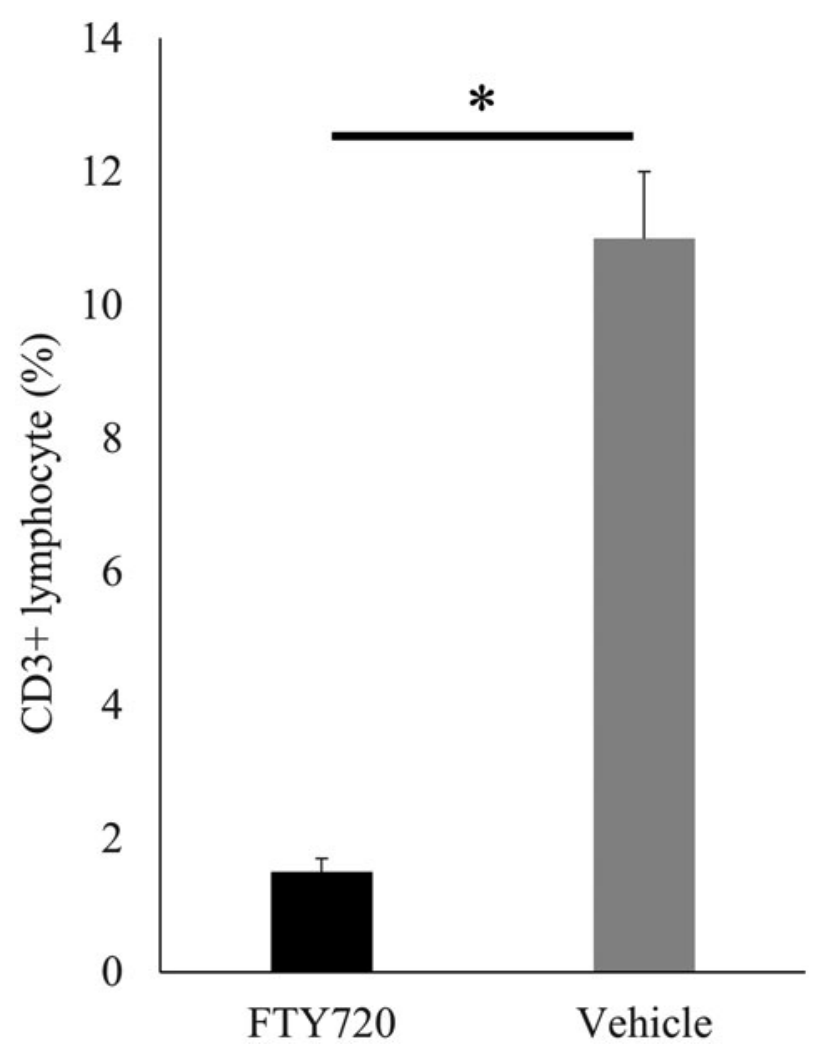

FIG. 3. Effect of FTY720 on systemic inflammation. Percentage of $\mathrm{CD}^{+} \mathrm{T}$ lymphocytes in peripheral blood measured using flow cytometry 3 days after the SCI. A significant decrease can be seen in the FTY720 group $(* p<0.01)$. SCI, spinal cord injury. 
A
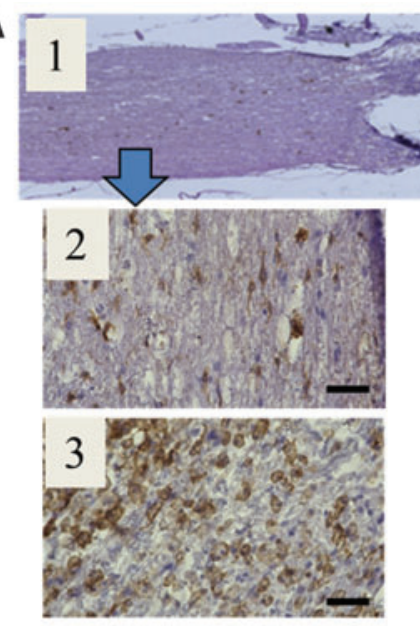

C

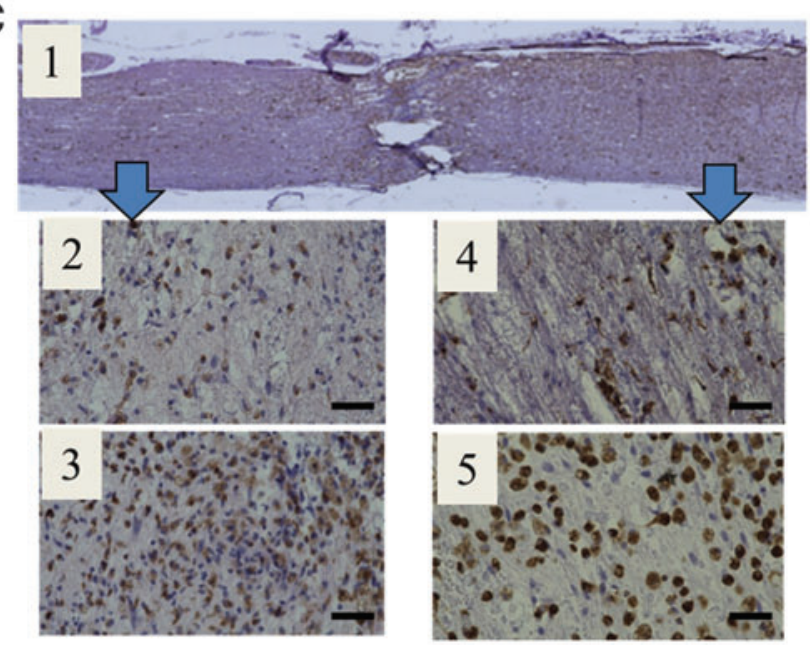

B

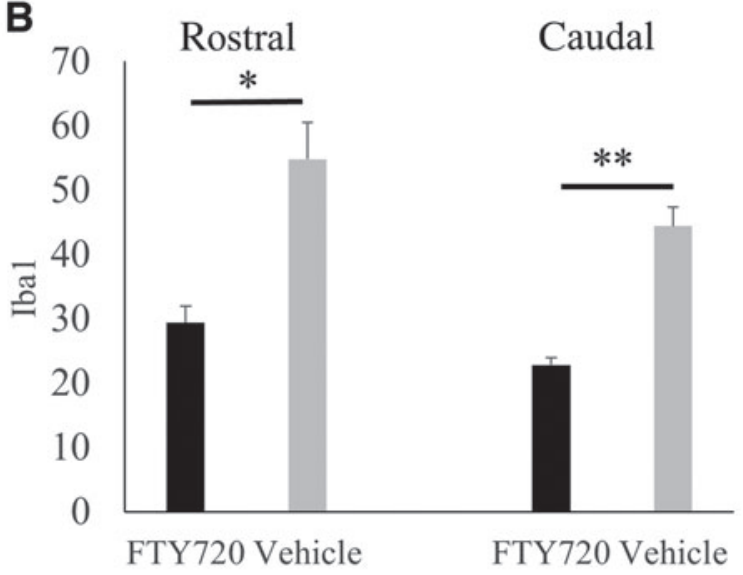

D

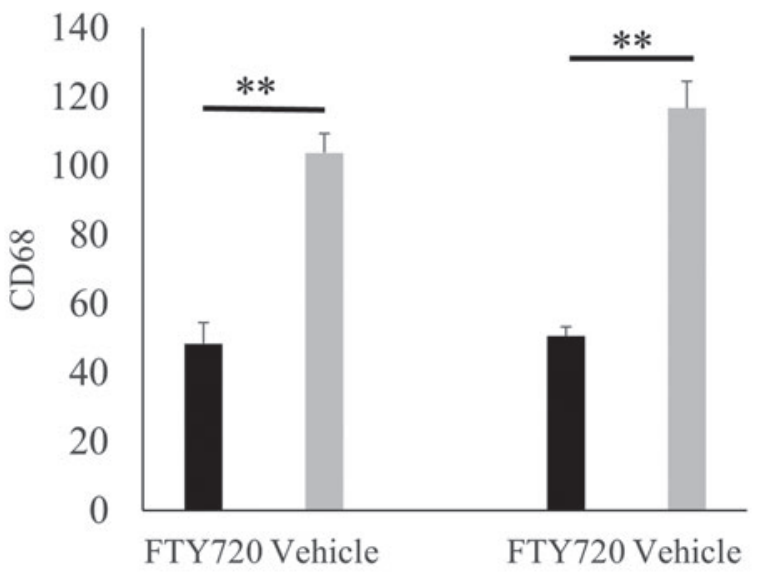

FIG. 4. Effect of FTY720 on local inflammatory cells. (A and B) Detection of activated microglia using Iba1 staining. (A-1) Representative images of Iba1 staining of the spinal cord 1 week after the SCI. In FTY720-treated rats, Iba1 staining is significantly reduced in both the rostral (A-2, FTY720; A-3, vehicle) and caudal (A-4, FTY720; A-5, vehicle) lesions. Scale bar=20 $\mu$ m. The FTY720-induced decrease in Ibal staining is significant in both the rostral $(* p<0.05)$ and caudal $(* * p<0.01)$ lesions (B). (C and D) Detection of activated macrophages using CD68 staining. (C-1) Representative images of CD68 staining of the spinal cord 1 week after the SCI. In FTY720-treated rats, CD68 staining is significantly reduced in both the rostral (C-2, FTY720; C-3, vehicle) and caudal (C-4, FTY720; C-5, vehicle) lesions. The FTY720-induced decrease in CD68 staining is significant in both the rostral and caudal lesions $(* * p<0.01 ; \mathrm{D}) . \mathrm{SCI}$, spinal cord injury.

\section{FTY720 induces systemic lymphopenia}

To check the systemic effects of FTY720, we evaluated the proportion of CD3-positive lymphocytes in the peripheral blood using flow cytometry 3 days after the SCI. FTY720-treated animals had a significantly lower proportion of $\mathrm{CD}^{+}$lymphocytes compared to vehicle-treated animals (Fig. 3).

\section{FTY720 decreased the extent of local inflammation and preserved the blood-brain barrier}

Local inflammation was investigated by monitoring both the activated microglia and infiltrated macrophages. Iba1 staining to detect activated microglia 1 week after the SCI showed significantly lower cell numbers at both the rostral and caudal ends of the damaged lesion in FTY720-treated animals when compared to vehicle-treated animals ( $p<0.01$ and 0.05 , respectively; Fig. 4A,B). Similarly, activation of macrophages measured by CD68 staining was also lower in FTY720-treated rats when compared to vehicle-treated rats (Fig. 4C,D; $p<0.01$ ).

Fibrin leakage, which is caused by dysregulation of the bloodbrain barrier and is associated with prolonged local inflammation, was also significantly lower in FTY720-treated animals when compared to the vehicle-treated group (Fig. 5A-C; $p<0.01$ ).

\section{FTY720 significantly reduced glial scars and preserved axons}

Glial scars were evaluated using GFAP staining. The area of GFAP-positive glial scars $4 \mathrm{~mm}$ caudal from the epicenter lesion was significantly smaller in FTY720-treated animals compared to that in vehicle-treated animals. FTY720 treatment resulted in an $80 \%$ reduction in the area of the glial scars (Fig. 6A-C). Residual axons were evaluated using FR dye. The dye was injected rostral to the damaged spinal cord, and the signal was obtained from the caudal end of the injured spinal cord, which enabled us to monitor 

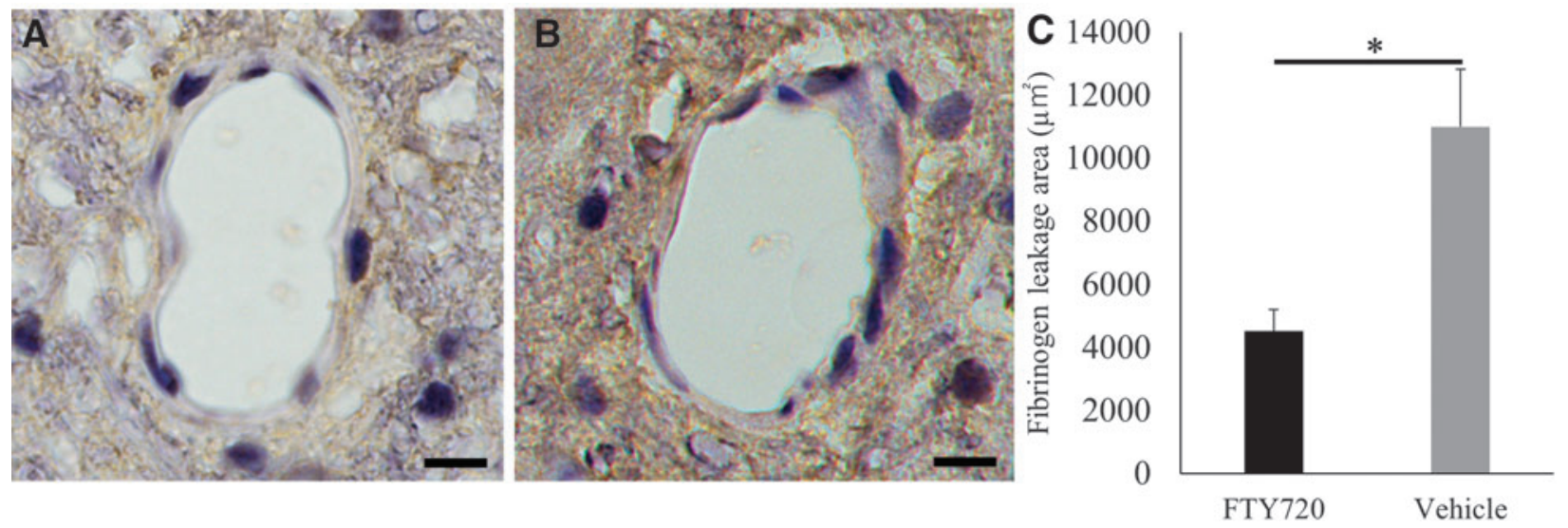

FIG. 5. FTY720 preserves blood-brain barrier integrity. Detection of fibrin leakage using fibrinogen staining. Representative images of fibrin leakage around a vessel in (A) FTY720-treated and (B) vehicle-treated animals. (C) FTY720 treatment significantly reduces fibrin leakage $\left({ }^{*} p<0.05\right.$; scale bar $\left.=10 \mu \mathrm{m}\right)$.

delivery of the FR dye along the residual axon across the damaged spinal cord. The number of FR-labeled axons within the dorsal funiculus in the dCST was higher in the FTY720 group, indicative of better preservation of the spinal axons (Fig. 6D-F).

\section{Evaluation of the sensory related nerve}

Expression of the sensory neurons and synapses was studied. Evaluation of CGRP at the dorsal horn for preservation of peripheral fibers showed no difference between the two groups (Fig. 7A-D). However, MOR, which is the interneuron for the local pain inhibitory synapse expression, was significantly preserved in lesions both rostral and caudal from the injury epicenter in FTY720-treated animals (Fig 7E-H). We further investigated the connectivity of the descending inhibitory pathway by HTT analysis. In the FTY720 groups, HTT was preserved in both the rostral and caudal lesions, whereas in the vehicle group it was significantly increased in the rostral lesion but decreased in the caudal lesion (Fig. 7I-L).
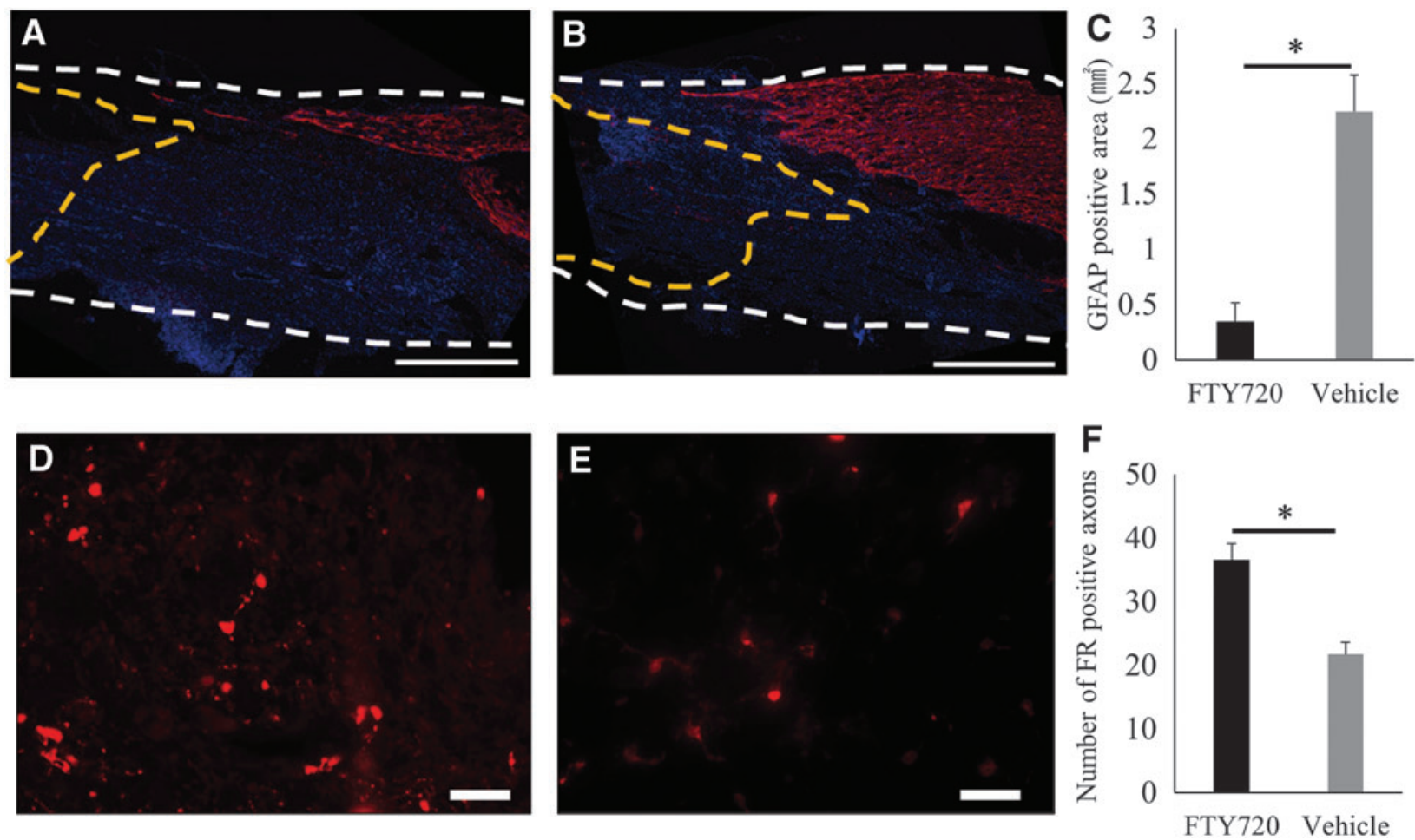

FIG. 6. FTY720 reduces glial scar formation and preserves axons. Evaluation of glial scars using GFAP staining. Representative images of glial scar formation in (A) FTY720-treated and (B) vehicle-treated rats. The white dotted line represents the spinal cord, and the yellow dotted line represents the epicenter of the lesion (scale bar $=1 \mathrm{~mm}$ ). (C) The glial scar area is significantly reduced in the FTY720-treated group when compared to that in the vehicle-treated group $\left({ }^{*} p<0.05\right)$. The connectivity of axons that runs through the injury area was evaluated using FR signals. The axonal network is significantly preserved in the FTY720-treated group (D) when compared to that in the vehicle-treated group (E). The number of FR-positive axons is significantly higher in the FTY720 group when compared to that in the vehicle-treated group $\left(\mathbf{F} ;{ }^{*} p<0.05\right.$; scale bar $\left.=50 \mu \mathrm{m}\right)$. FR, Fluoro-Ruby; GFAP, glial fibrillary acidic protein. 
A

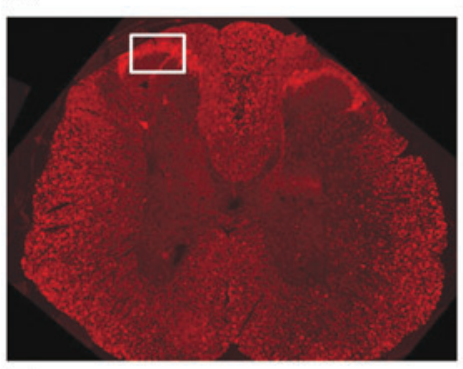

E

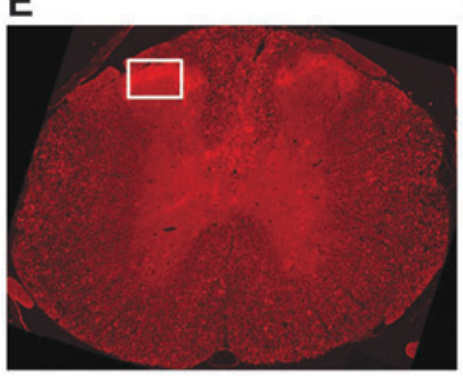

I

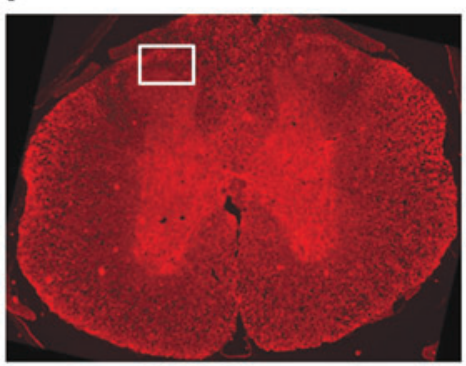

B

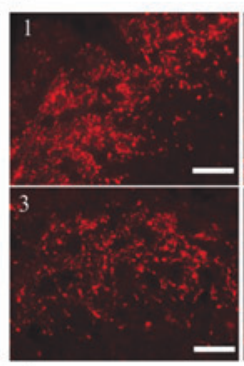

$F$

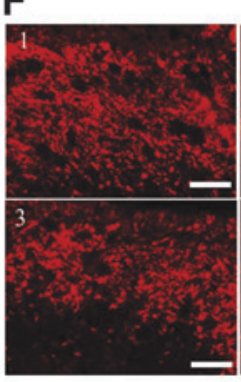

J

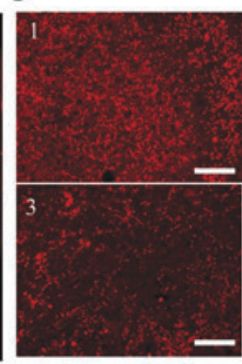

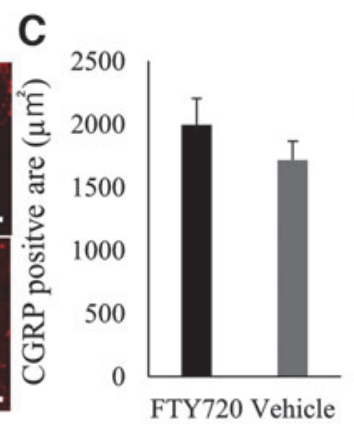

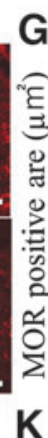

K
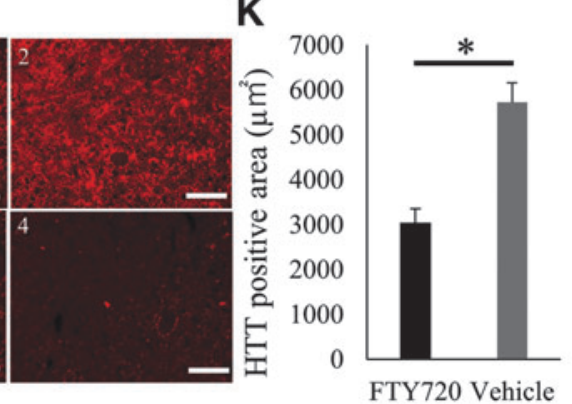
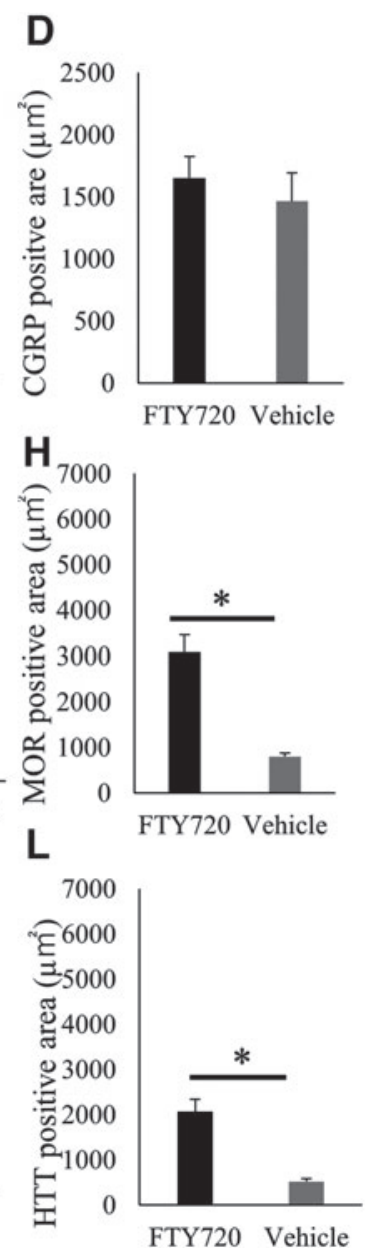

FIG. 7. Evaluation of sensory-related nerves and synapses. (A) Expression of calcitonin gene-related peptide (CGRP) at the dorsal horn of both rostral and caudal sides of the injury site. (B) No difference is seen in expression between the FTY720-treated (B1, rostral; B3, caudal) and vehicle-treated (B2, rostral; B4, caudal) groups in both the (C) rostral and (D) caudal lesions. (E) $\mu$ opioid receptor (MOR) expression is preserved in FTY720 rats in (F) both the (F1) rostral and (F3) caudal sides of the injured area compared to that in the vehicle group (rostral, F2; caudal, F4; rostral, G; caudal, H). (I) Expression of hydroxytryptamine transporter (HTT) is preserved in (J) both the (J1) rostral and (J3) caudal sides of the injury site in FTY720-treated animals. An increase in the HTT signal on the (J2) rostral side and decrease on the (J4) caudal side of the injury can be seen in the vehicle-treated group (rostral, K; caudal, L; ${ }^{*} p<0.05$; scale $\operatorname{bar}=20 \mu \mathrm{m})$.

\section{Discussion}

FTY720 was first synthesized from the fungal metabolite myriocin isolated from Isaria sinclairii. Its strong immunosuppressive potential led to its approval as the first oral immunomodulatory drug for MS. ${ }^{11}$ Phosphorylation of FTY720 to its active form, FTY720-P, is catalyzed by sphingosine kinase, and FTY720-P shows unique biological effects as a functional agonist and antagonist through S1P receptors. FTY720 acts as an agonist by binding to four of the five known G-protein-coupled S1P receptors $\left(\mathrm{S}_{1} \mathrm{P}_{1}\right.$, $\mathrm{S}_{1} \mathrm{P}_{3-5}$ ) expressed on the membrane surfaces of cells, including neurons, astrocytes, and vessel endothelial cells. These pathways regulate multiple cellular events, including cell proliferation and survival, mostly in a cell-protective manner. FTY720 acts as an antagonist by the temporary downregulation and degradation of $\mathrm{S} 1 \mathrm{P}$ receptors on lymphocytes, preventing their entry into the bloodstream from the lymph nodes, resulting in strong systemic lymphopenia. Because of its agonistic and antagonistic properties, FTY720 has been increasingly recognized as a potential therapeutic target for neurological diseases, including neurodegenerative diseases, stroke, traumatic brain injury, and SCI.

Previous studies have shown that administration of FTY720 in the acute phase of SCI promotes better functional recovery, including hindlimb motor and bladder functions, by reducing inflammation and, possibly, secondary damage. ${ }^{9,12,32}$ Consistent with these reports, we found that in FTY720-treated rats, the fluorescent axon tracer injected at the rostral side of the injury was highly detectable at the caudal side, indicating the preservation and/or regeneration of the descending axonal fibers. Together with these results, we show that FTY720 can attenuate neuropathic pain after SCI.

Given that the pathophysiology of neuropathic pain after SCI is not fully understood, many of our findings from this study may be helpful in understanding the mechanism of pain alleviation. First, lymphopenia, followed by lower local inflammation, may lead to less secondary damage to neuronal networks. We found that, 3 days after SCI, lymphocyte counts were significantly lower in the FTY720 group than in the vehicle group. Moreover, in FTY720treated rats, activated microglia and macrophages on both the 
rostral and caudal sides of the injury site were significantly decreased 7 days after the injury. This is unlikely to be a direct effect of FTY720 on the microglia and macrophages given that these cells mostly express $\mathrm{S}_{1} \mathrm{P}_{2}$ receptors, to which FTY720 does not directly bind. ${ }^{11,33}$ We therefore believe that the decrease in systemic inflammation could have led to an attenuation of the secondary local inflammation. Second, preservation of the blood-brain barrier integrity can also decrease secondary damage. FTY720 has been shown to protect tight and adherence junctions through $\mathrm{S} 1 \mathrm{P}$ receptors. ${ }^{34}$

We also found lower fibrin leakage around blood vessels in FTY720-treated animals. The blood components leaking though the blood-brain barrier have been shown to increase activation of microglia, damage to endothelial cells, and penetration of blood macrophages into the damaged area. Third, FTY720 has been shown to suppress the activation of astrocytes, thereby reducing astrogliosis after central nervous system disorders, ${ }^{8-10}$ which is consistent with our finding of reduced gliosis. In FTY720-treated animals, with lower local inflammation and gliosis, there was a higher chance for the damaged neuronal network to survive and recover, allowing neuronal regeneration, ultimately resulting in better neurological recovery.

One of the novel findings in this report is that FTY720 can ameliorate neuropathic pain through preservation of the descending inhibitory pain pathway. Bruce and colleagues have reported that disruption of the descending antinociceptive serotonergic tracts is responsible for neuropathic pain in the SCI model. ${ }^{29} \mathrm{We}$ also found that although the SCI did not change the distribution of CGRP in the dorsal horn, serotonin immunoreactivity was increased at the rostral side, but decreased at the caudal sides of the injury in vehicletreated rats. Bruce and colleagues concluded that the descending pain inhibitory tract is blocked by SCI, which results in decreased serotonergic immunoreactivity caudal of SCI, resulting in positive feedback to the rostral side of the SCI, thereby upregulating the descending pain inhibitory pathway. FTY720 has also been reported to be effective against neuropathic pain in a peripheral nerve injury model ${ }^{35}$, but its effects on SCI-related neuropathic pain are not well elucidated. In this study, we have shown that FTY720 prevents degradation of the descending inhibitory pain pathway and MOR, which is the local interneuron for controlling pain, but did not observe any effect on the peripheral $\mathrm{C}$ fiber.

FTY720 has been reported to mediate antinociceptive effects by reducing prostaglandin synthesis through inhibition of cytosolic phospholipase A2, which accounts for central pain. ${ }^{36,37}$ We believe that FTY720 inhibits progression of the adhesive arachnoiditis as observed by the lower gliosis in the chronic phase of the injury, which could also account for better pain recovery. However, the pain and inflammation cascade are intricately inter-related, and further investigation is warranted to elucidate the underlying mechanisms. ${ }^{38}$

Our study has several limitations. First, we were not able to directly connect systemic inflammation to local inflammation. Given that activated lymphocytes secrete several proinflammatory factors, including interleukin-3 and nuclear factor kappa-lightchain-enhancer of activated B cells, which are thought to upregulate microglia and macrophages, FTY720 can also directly inhibit microglial activation. Gene knockout mouse models or models in which leukocytes are abolished might help in determining the effects of FTY720 more precisely.

Second, we did not include other types of SCI models in this study. Transection injury and crush injury models are also examined for SCI study experiments. Each of these models is important to study different aspects of the pathophysiology of SCI; however, because of improvements in car safety, the instances of complete SCI are decreasing, whereas with the increase in the aging popu- lation, the number of elderly individuals with incomplete SCI is increasing, and a compression model would be most suitable for evaluating clinical settings.

Third, we were also not able to examine the route- and dosedependent therapeutic effects of FTY720, which will be crucial for determining its optimal dose for clinical use. Oral intake of FTY720 should also be considered, given that FTY720 administration is approved through the oral route. Intraperitoneal injection was chosen in this study, because SCI can cause severe illness and apathy in animals for at least a week, which makes it difficult to monitor whether they have ingested the appropriate amount of FTY720. Autonomic dysregulation after SCI can lead to bowel disfunction, and this may cause heterogenous absorption rates of FTY720 among animals. We considered that intraperitoneal injections would overcome these problems.

Our results here provide new insight into regenerative medicine by the administration of FTY720 in the acute phase of SCI. FTY720 ameliorates inflammatory and glial scar formation, thereby providing an optimal environment for administration of stem cells for regeneration in the subacute phase of SCI.

In conclusion, acute administration of FTY720 after SCI can ameliorate neuropathic pain, presumably by reducing systemic and local inflammation, maintaining blood-brain barrier integrity, and reducing glial scar, thereby preserving the descending inhibitory pain pathway.

\section{Funding Information}

This study was supported by AMED under Grant Number JP17bk0104045, and Japan Society for the Promotion of Science Fujita Memorial Fund for Medical Research.

\section{Author Disclosure Statement}

No competing financial interests exist.

\section{References}

1. van den Berg, M.E., Castellote, J.M., Mahillo-Fernandez, I., and de Pedro-Cuesta, J. (2010). Incidence of spinal cord injury worldwide: a systematic review. Neuroepidemiology 34, 184-192; discussion, 192.

2. Finnerup, N.B., Norrbrink, C., Trok, K., Piehl, F., Johannesen, I.L., Sorensen, J.C., Jensen, T.S., and Werhagen, L. (2014). Phenotypes and predictors of pain following traumatic spinal cord injury: a prospective study. J. Pain 15, 40-48.

3. Siddall, P.J., McClelland, J.M., Rutkowski, S.B., and Cousins, M.J. (2003). A longitudinal study of the prevalence and characteristics of pain in the first 5 years following spinal cord injury. Pain 103, 249-257.

4. Stormer, S., Gerner, H.J., Gruninger, W., Metzmacher, K., Follinger, S., Wienke, C., Aldinger, W., Walker, N., Zimmermann, M., and Paeslack, V. (1997). Chronic pain/dysaesthesiae in spinal cord injury patients: results of a multicentre study. Spinal Cord 35, 446-455.

5. Jensen, M.P., Kuehn, C.M., Amtmann, D., and Cardenas, D.D. (2007). Symptom burden in persons with spinal cord injury. Arch. Phys. Med. Rehabil. 88, 638-645.

6. Wilson, J.R., Forgione, N., and Fehlings, M.G. (2013). Emerging therapies for acute traumatic spinal cord injury. CMAJ 185, 485-492.

7. Fujita, T., Inoue, K., Yamamoto, S., Ikumoto, T., Sasaki, S., Toyama, R., Chiba, K., Hoshino, Y., and Okumoto, T. (1994). Fungal metabolites. Part 11. A potent immunosuppressive activity found in Isaria sinclairii metabolite. J. Antibiot. (Tokyo) 47, 208-215.

8. Choi, J.W., Gardell, S.E., Herr, D.R., Rivera, R., Lee, C.W., Noguchi, K., Teo, S.T., Yung, Y.C., Lu, M., Kennedy, G., and Chun, J. (2011). FTY720 (fingolimod) efficacy in an animal model of multiple sclerosis requires astrocyte sphingosine 1-phosphate receptor 1 (S1P1) modulation. Proc. Natl. Acad. Sci. U. S. A. 108, 751-756.

9. Wang, J., Wang, J., Lu, P., Cai, Y., Wang, Y., Hong, L., Ren, H., Heng, B.C., Liu, H., Zhou, J., and Ouyang, H. (2015). Local delivery of FTY720 in PCL membrane improves SCI functional recovery by reducing reactive astrogliosis. Biomaterials 62, 76-87. 
10. Foster, C.A., Howard, L.M., Schweitzer, A., Persohn, E., Hiestand, P.C., Balatoni, B., Reuschel, R., Beerli, C., Schwartz, M., and Billich, A. (2007). Brain penetration of the oral immunomodulatory drug FTY720 and its phosphorylation in the central nervous system during experimental autoimmune encephalomyelitis: consequences for mode of action in multiple sclerosis. J. Pharmacol. Exp. Ther. 323, 469-475.

11. Kawabori, M., Kacimi, R., Karliner, J.S., and Yenari, M.A. (2013). Sphingolipids in cardiovascular and cerebrovascular systems: pathological implications and potential therapeutic targets. World J. Cardiol. 5, 75-86.

12. Lee, K.D., Chow, W.N., Sato-Bigbee, C., Graf, M.R., Graham, R.S., Colello, R.J., Young, H.F., and Mathern, B.E. (2009). FTY720 reduces inflammation and promotes functional recovery after spinal cord injury. J. Neurotrauma 26, 2335-2344.

13. Norimatsu, Y., Ohmori, T., Kimura, A., Madoiwa, S., Mimuro, J., Seichi, A., Yatomi, Y., Hoshino, Y., and Sakata, Y. (2012). FTY720 improves functional recovery after spinal cord injury by primarily nonimmunomodulatory mechanisms. Am. J. Pathol. 180, 1625-1635.

14. Austin, J.W., Kang, C.E., Baumann, M.D., DiDiodato, L., Satkunendrarajah, K., Wilson, J.R., Stanisz, G.J., Shoichet, M.S., and Fehlings, M.G. (2012). The effects of intrathecal injection of a hyaluronanbased hydrogel on inflammation, scarring and neurobehavioural outcomes in a rat model of severe spinal cord injury associated with arachnoiditis. Biomaterials 33, 4555-4564.

15. Seki, T., and Fehlings, M.G. (2008). Mechanistic insights into posttraumatic syringomyelia based on a novel in vivo animal model. Laboratory investigation. J. Neurosurg. Spine 8, 365-375.

16. Austin, J.W., Afshar, M., and Fehlings, M.G. (2012). The relationship between localized subarachnoid inflammation and parenchymal pathophysiology after spinal cord injury. J. Neurotrauma 29, 1838 1849.

17. Chiba, Y., Kuroda, S., Osanai, T., Shichinohe, H., Houkin, K., and Iwasaki, Y. (2012). Impact of ageing on biological features of bone marrow stromal cells (BMSC) in cell transplantation therapy for CNS disorders: functional enhancement by granulocyte-colony stimulating factor (G-CSF). Neuropathology 32, 139-148.

18. Hao, J.X., Xu, X.J., Aldskogius, H., Seiger, A., and Wiesenfeldhallin, Z. (1991). Allodynia-like effects in rat after ischemic spinal-cord injury photochemically induced by laser irradiation. Pain 45, 175-185.

19. Xu, X.J., Hao, J.X., Aldskogius, H., Seiger, A., and WiesenfeldHallin, Z. (1992). Chronic pain-related syndrome in rats after ischemic spinal cord lesion: a possible animal model for pain in patients with spinal cord injury. Pain 48, 279-290.

20. Basso, D.M., Beattie, M.S., and Bresnahan, J.C. (1995). A sensitive and reliable locomotor rating scale for open field testing in rats. J. Neurotrauma 12, 1-21.

21. Shono, Y., Toubai, T., Ota, S., Ibata, M., Mashiko, S., Hirate, D., Miura, Y., Umehara, S., Toyoshima, N., Tanaka, J., Asaka, M., and Imamura, M. (2006). Abnormal expansion of naive B lymphocytes after unrelated cord blood transplantation-a case report. Clin. Lab. Haematol. 28, 351-354.

22. Seki, T., Hida, K., Tada, M., Koyanagi, I., and Iwasaki, Y. (2002). Graded contusion model of the mouse spinal cord using a pneumatic impact device. Neurosurgery 50, 1075-1081; discussion, 1081-1072.

23. Dohrmann, G.J., and Panjabi, M.M. (1976). "Standardized" spinal cord trauma: biomechanical parameters and lesion volume. Surg. Neurol. 6, 263-267.

24. Johnson, V.E., Weber, M.T., Xiao, R., Cullen, D.K., Meaney, D.F., Stewart, W., and Smith, D.H. (2018). Mechanical disruption of the blood-brain barrier following experimental concussion. Acta Neuropathol. 135, 711-726.

25. Kawabori, M., Hokari, M., Zheng, Z., Kim, J.Y., Calosing, C., Hsieh, C.L., Nakamura, M.C., and Yenari, M.A. (2013). Triggering receptor expressed on myeloid cells-2 correlates to hypothermic neuroprotection in ischemic stroke. Ther. Hypothermia Temp. Manag. 3, 189-198.

26. Kawabori, M., Kacimi, R., Kauppinen, T., Calosing, C., Kim, J.Y., Hsieh, C.L., Nakamura, M.C., and Yenari, M.A. (2015). Triggering receptor expressed on myeloid cells 2 (TREM2) deficiency attenuates phagocytic activities of microglia and exacerbates ischemic damage in experimental stroke. J. Neurosci. 35, 3384-3396.

27. Kudo, T., Konno, K., Uchigashima, M., Yanagawa, Y., Sora, I., Minami, M., and Watanabe, M. (2014). GABAergic neurons in the ventral tegmental area receive dual GABA/enkephalin-mediated inhibitory inputs from the bed nucleus of the stria terminalis. Eur. J. Neurosci. 39, 1796-1809.

28. Somogyi, J., Baude, A., Omori, Y., Shimizu, H., El Mestikawy, S., Fukaya, M., Shigemoto, R., Watanabe, M., and Somogyi, P. (2004). GABAergic basket cells expressing cholecystokinin contain vesicular glutamate transporter type 3 (VGLUT3) in their synaptic terminals in hippocampus and isocortex of the rat. Eur. J. Neurosci. 19, 552-569.

29. Bruce, J.C., Oatway, M.A., and Weaver, L.C. (2002). Chronic pain after clip-compression injury of the rat spinal cord. Exp. Neurol. 178, 33-48.

30. Chiba, Y., Kuroda, S., Maruichi, K., Osanai, T., Hokari, M., Yano, S., Shichinohe, H., Hida, K., and Iwasaki, Y. (2009). Transplanted bone marrow stromal cells promote axonal regeneration and improve motor function in a rat spinal cord injury model. Neurosurgery 64, 991-999; discussion, 999-1000.

31. Chuah, M.I., Choi-Lundberg, D., Weston, S., Vincent, A.J., Chung, R.S., Vickers, J.C., and West, A.K. (2004). Olfactory ensheathing cells promote collateral axonal branching in the injured adult rat spinal cord. Exp. Neurol. 185, 15-25.

32. Zhang, J., Zhang, A., Sun, Y., Cao, X., and Zhang, N. (2009). Treatment with immunosuppressants FTY720 and tacrolimus promotes functional recovery after spinal cord injury in rats. Tohoku $\mathrm{J}$. Exp. Med. 219, 295-302.

33. Sapkota, A., Gaire, B.P., Kang, M.G., and Choi, J.W. (2019). S1P2 contributes to microglial activation and M1 polarization following cerebral ischemia through ERK1/2 and JNK. Sci. Rep. 9, 12106.

34. Czech, B., Pfeilschifter, W., Mazaheri-Omrani, N., Strobel, M.A., Kahles, T., Neumann-Haefelin, T., Rami, A., Huwiler, A., and Pfeilschifter, J. (2009). The immunomodulatory sphingosine 1-phosphate analog FTY720 reduces lesion size and improves neurological outcome in a mouse model of cerebral ischemia. Biochem. Biophys. Res. Commun. 389, 251-256.

35. Sim-Selley, L.J., Wilkerson, J.L., Burston, J.J., Hauser, K.F., McLane, V., Welch, S.P., Lichtman, A.H., and Selley, D.E. (2018). Differential tolerance to FTY720-induced antinociception in acute thermal and nerve injury mouse pain models: role of sphingosine-1-phosphate receptor adaptation. J. Pharmacol. Exp. Ther. 366, 509-518.

36. Coste, O., Pierre, S., Marian, C., Brenneis, C., Angioni, C., Schmidt, H., Popp, L., Geisslinger, G., and Scholich, K. (2008). Antinociceptive activity of the S1P-receptor agonist FTY720. J. Cell. Mol. Med. 12, 995-1004.

37. Zhang, D.D., Linke, B., Suo, J., Zivkovic, A., Schreiber, Y., Ferreiros, N., Henke, M., Geisslinger, G., Stark, H., and Scholich, K. (2015). Antinociceptive effects of FTY720 during trauma-induced neuropathic pain are mediated by spinal S1P receptors. Biol. Chem. 396, 783-794.

38. Nees, T.A., Finnerup, N.B., Blesch, A., and Weidner, N. (2017). Neuropathic pain after spinal cord injury: the impact of sensorimotor activity. Pain 158, 371-376.

Address correspondence to: Masahito Kawabori, MD Department of Neurosurgery Hokkaido University Graduate School of Medicine Kita 15, Nishi 7, Kita-ku Sapporo, Hokkaido 060-8638 Japan E-mail: masahitokawabori@yahoo.co.jp 